Mens

revue d'histoire intellectuelle de l'Amérique française

\title{
Littérature personnelle et histoire de la lecture : de la théorie à la pratique
}

\section{Cécile Facal}

Volume 5, numéro 2, printemps 2005

L'histoire du livre au Québec, de la Nouvelle-France au XX ${ }^{\mathrm{e}}$ siècle

URI : https://id.erudit.org/iderudit/1024355ar

DOI : https://doi.org/10.7202/1024355ar

Aller au sommaire du numéro

Éditeur(s)

Centre de recherche en civilisation canadienne-française

ISSN

1492-8647 (imprimé)

1927-9299 (numérique)

Découvrir la revue

Citer cet article

Facal, C. (2005). Littérature personnelle et histoire de la lecture : de la théorie à la pratique. Mens, 5(2), 215-239. https://doi.org/10.7202/1024355ar

\section{Résumé de l'article}

Les pratiques individuelles de lecture sont relativement peu étudiées en comparaison des pratiques collectives. Les textes de littérature personnelle constituent des sources essentielles permettant d'accéder aux lecteurs individuels. Leur utilisation et le traitement des informations qu'ils fournissent ne vont cependant pas de soi. Dans cet article, qui se fonde sur les résultats de l'étude d'une vingtaine de textes personnels d'écrivains québécois, une série d'observations pratiques concernant le maniement des sources personnelles et leurs possibilités opératoires s'articulent à des remarques plus générales sur les recherches actuelles autour de la lecture. Il s'agit donc d'identifier quels sont les risques et les promesses de l'exploitation des corpus de littérature personnelle pour une meilleure connaissance des pratiques de lecture. 


\title{
LITTÉRATURE PERSONNELLE ET HISTOIRE DE LA LECTURE : DE LA THÉORIE À LA PRATIQUE
}

\author{
Cécile Facal \\ Département de langue et littérature françaises \\ Université McGill \\ cecilefacal@yahoo.ca
}

\section{Résumé}

Les pratiques individuelles de lecture sont relativement peu étudiées en comparaison des pratiques collectives. Les textes de littérature personnelle constituent des sources essentielles permettant d'accéder aux lecteurs individuels. Leur utilisation et le traitement des informations qu'ils fournissent ne vont cependant pas de soi. Dans cet article, qui se fonde sur les résultats de l'étude d'une vingtaine de textes personnels d'écrivains québécois, une série d'observations pratiques concernant le maniement des sources personnelles et leurs possibilités opératoires s'articulent à des remarques plus générales sur les recherches actuelles autour de la lecture. Il s'agit donc d'identifier quels sont les risques et les promesses de l'exploitation des corpus de littérature personnelle pour une meilleure connaissance des pratiques de lecture.

\section{Abstract}

Individual reading practices have received little scholarly attention when compared to collective practices. The study of personal literature, moreover, is essential to gaining an insight into individual readers. However, the methods required to 
analyse personal literature are not readily apparent. This article, which draws on the results of a study of twenty personal texts written by Quebec writers, contains a series of practical observations relating to the use and analysis of personal sources and some more general considerations regarding recent research on reading. The author identifies the risks and rewards of studying personal literature to gain a better understanding of reading practices.

Les recherches sur la lecture dans le domaine de l'histoire du livre et de l'imprimé se développent depuis plusieurs années déjà et offrent un complément essentiel à celles portant sur l'histoire de la production matérielle, de l'édition et de la diffusion du livre. Une récente bibliographie des études sur la question révèle que la majorité d'entre elles s'intéressent aux pratiques collectives de lecture ${ }^{1}$. Pour une grande part, ces recherches utilisent les mêmes sources que l'histoire du livre qu'elles interrogent cependant du point de vue du lecteur. Le large éventail de ces sources s'étend du catalogue de bibliothèque ou de librairie à la présentation matérielle des livres et aux textes prescriptifs sur la manière de lire; chacune permet de mesurer et de qualifier des aspects de la lecture aussi divers que la censure, les niveaux d'alphabétisation ou l'offre de livres ${ }^{2}$. Ces objets d'étude, s'ils touchent au cœur de la lecture entendue au sens large comme phénomène social, demeurent très éloignés de l'acte de lecture ${ }^{3}$. Les pratiques individuelles de lecture sont d'une certaine manière insaisissables. Contrairement à l'écriture, elles demeurent « toujours dans l'ordre de l'éphémère [...], s'éparpille[nt] en une infinité d'actes singuliers, [...] s'affranchi[ssent] volontiers de toutes les contraintes voulant [les] soumettre ${ }^{4}$ ». Leur intérêt n'en est pas moins indéniable : en sémiotique, en pédagogie, en littérature, la réflexion théorique se poursuit depuis des années afin d'éclairer l'acte de lecture, de distinguer diverses manières de lire et d'expliquer les mécanismes de production 
du sens. D'un point de vue historique, la lecture individuelle, dont il n'est plus nécessaire de démontrer l'historicité, constitue un objet aussi prometteur que problématique et sans doute l'un des défis les plus imposants qui s'offrent aux historiens du livre, à cause justement de sa fugacité.

Afin d'accéder de façon relativement directe aux lecteurs singuliers, l'une des sources les plus riches est, selon plusieurs, la littérature personnelle. On y trouverait d'abord des titres de textes et des indications sur les circonstances de leur lecture - les lieux et moments où elle s'exerce, sa fréquence, sa rapidité ou sa lenteur. Elle serait également susceptible de révéler, du moins en partie, ses motivations, ses objectifs et ses effets intellectuels ou émotifs sur le lecteur. Plusieurs études de cas qui s'appuient sur des documents d'archives aussi rares qu'exceptionnels ont dévoilé les traits distinctifs de lecteurs et ont permis d'esquisser des généralisations sur les pratiques de lecture d'une époque. La correspondance de Jean Ranson a permis d'établir les particularités d'une lecture "rousseauiste ${ }^{6} »$; le journal intime d'Anna Larpent témoigne de la variété des façons de lire chez un même individu ${ }^{7}$; les mémoires de Valentin Jameray-Duval présentent la lecture d'un autodidacte ${ }^{8}$; dans le domaine québécois, LouisJoseph, Amédée et Lactance Papineau ainsi que Joséphine Marchand-Dandurand se révèlent à travers leur correspondance et leurs journaux intimes?.

Dans le sillage de telles études, l'analyse d'une vingtaine de textes personnels d'écrivains québécois, entreprise dans le cadre d'un projet de recherche sur les pratiques de lecture chez les intellectuels du XX $\mathrm{XX}^{\mathrm{e}}$ siècle ${ }^{10}$, nous amène à nuancer le postulat selon lequel les sources personnelles permettent un accès direct aux individus lecteurs. La littérature personnelle ne peut être toujours considérée comme un document de première main sur les pratiques de lecture. Ses réfé- 
rences à la lecture sont si souvent incomplètes qu'une interprétation fructueuse n'est possible qu'à condition de s'appuyer sur une variété de sources. En outre, suivant la fonction du texte et selon qu'il est ou non destiné à la publication, il arrive que la main de la rhétorique lui imprime une marque dont le chercheur doit tenir compte. Une série d'observations pratiques concernant le maniement des sources personnelles et leurs possibilités opératoires s'articuleront donc ici à des remarques plus générales sur les recherches actuelles autour de la lecture ${ }^{11}$. En effet, il nous paraît essentiel, en bonne méthode, d'identifier quels sont les risques et les promesses d'une telle entreprise pour la meilleure connaissance des pratiques de lecture.

\section{Des sources de valeur inégale}

Dans le cadre d'une exploration qui s'interroge sur les pratiques de lecture, c'est-à-dire sur leur comment et leur pourquoi, l'identification des passages où elles sont représentées directement constitue une première étape. Nous avons donc porté notre attention, d'une part, sur les discours traitant explicitement de la lecture (et non, dans un premier temps, des textes lus), et d'autre part, sur les passages où la lecture est décrite ou mise en scène. Ces derniers permettent d'élucider les circonstances pratiques de la lecture, identifient les conditions qui la facilitent ou l'entravent, et témoignent des éventuels effets des textes sur les lecteurs. Contre toute attente, les résultats de ce premier relevé s'avèrent mitigés, en dépit de la position privilégiée des écrivains en ce qui touche la lecture.

Dans un tel corpus, des mines d'or retiennent tout de même l'attention. Le journal et la correspondance d'Hector de Saint-Denys Garneau ${ }^{12}$ contiennent de nombreuses pages constituées de notes de lecture et de commentaires sur les 
textes, sur les auteurs ou sur les sentiments ressentis lors de la lecture. Ils permettent de reconstituer les exigences du poète vis-à-vis les textes et la relation d'admiration ou de quasifraternité établie avec certains écrivains. Tout aussi riche, le Journal dénoué du poète Fernand Ouellette ${ }^{13}$, essai autobiographique composé rétrospectivement à partir de son journal intime, raconte à travers le filtre des lectures le parcours affectif et intellectuel menant l'auteur à la découverte de soi et au développement de son identité poétique ${ }^{14}$. À l'opposé, certains textes restent pratiquement muets quant aux lectures de leur auteur. C'est le cas des Mémoires intimes de Louis Fréchette $^{15}$, desquels la figure du livre est absente même si l'auteur développe longuement l'importance des conteurs et de la tradition orale dans un monde d'illettrés et ironise sur l'inanité de l'enseignement primaire qu'il a reçu.

La plupart des textes étudiés se situent à mi-chemin entre ces deux cas de figure. Plusieurs contiennent quelques pages éloquentes sur la découverte de la lecture dans l'enfance, font état du contrôle des lectures dans les collèges et des moyens ayant permis aux auteurs d'y échapper. Ceux qui ont grandi hors des centres urbains ou dans des milieux modestes s'accordent pour déplorer le manque de disponibilité du livre. D'aucuns font œuvre utile en dressant une liste de leurs principales influences littéraires. Victor Barbeau jette un coup d'œil critique, dans un chapitre intitulé "Lectures ", aux ouvrages ayant reçu durant sa jeunesse la faveur du public et parfois de la critique, «idoles d'argile » retombées depuis longtemps dans l'ombre au moment de la publication de La tentation du passé ${ }^{16}$. Entre le médiocre "déchet " et des œuvres de plus de valeur, l'autobiographe établit ici un partage et revisite des jugements passés, les siens comme ceux de l'opinion, fournissant ainsi des indications sur deux moments distincts de son parcours de lecteur, le temps de sa 
jeunesse et celui de l'écriture. Roger Duhamel rappelle lui aussi les auteurs admirés durant les années de collège - Gide, Mauriac, Montherlant, Lacretelle, Duhamel, Julien Green, Bernanos, Jules Romains, Daniel-Rops - et rouvre le dossier contenant ses notes de lecture pour rappeler en quoi ces auteurs touchaient son cœur d'adolescent ${ }^{17}$. Jean-Paul Desbiens donne le palmarès de ses auteurs ${ }^{18}$ et de ses romans préférés $^{19}$. En somme, les représentations directes de la lecture offertes dans ces textes personnels sont isolées malgré leur richesse. Elles le sont du moins en proportion de l'abondance de citations, de références et d'allusions littéraires qui s'y trouvent par ailleurs. Les textes sont en effet truffés de références intertextuelles témoignant d'une culture du livre qui elle-même constitue une trace des lectures, sans que celles-ci soient systématiquement évoquées.

Il en est ainsi du Journal de Ringuet ${ }^{20}$, dont la seule partie intégralement conservée a été rédigée durant les années 1920, lors d'une série de séjours à Paris comme stagiaire en médecine, séjours entrecoupés de voyages dans toute la France, en Belgique, en Italie et en Allemagne. Cela en fait une sorte de journal de voyage "composé à tête reposée à partir de notes prises sur le vif ${ }^{21}$. En voyage, Ringuet semble ne pas s'encombrer de livres, si ce n'est d'un guide - le Baedeker est plus d'une fois mentionné. Cependant, la culture livresque de l'auteur s'anime au contact des sites visités. Tantôt un lieu rappelle les origines ou les voyages d'un écrivain: Chateaubriand est évoqué lors d'un séjour en Bretagne, la figure de Dante apparaît à Ravenne où se trouve son tombeau, telle rue ou telle église parisienne rappelle Huysmans $\mathrm{s}^{22}$ : alors on a l'impression qu'allusion est faite, plus qu'aux textes de ces auteurs, aux personnages qu'ils représentent pour Ringuet, et cependant rien ne peut nous assurer de sa lecture de leurs textes. Ailleurs, des lieux font surgir de la mémoire de l'auteur 
des vers, des phrases ou des idées tirées d'œuvres citées plus ou moins directement, identifiées de manière plus ou moins explicite : parfois seul le nom d'un auteur est mentionné, d'autres fois, les italiques ou les guillemets nous indiquent une citation possible. Quant à la pratique de la lecture, elle apparaît peu. En passant, l'auteur rappelle avoir feuilleté un livre pour tromper l'attente, entre autres sur le bateau qui l'emmène vers l'Europe; il évoque brièvement ses séances de bouquinerie aux quais, et guère plus d'une ou deux phrases laissent entrevoir ses lectures d'études.

\section{2. Élargir le champ : références, citations, critiques}

Les textes personnels, s'ils ne constituent pas systématiquement une source de renseignements sur les pratiques de lecture de leurs auteurs ou de leurs contemporains, restent toutefois dignes d'intérêt par la place prépondérante qu'y occupe la littérature. L'irrégularité des résultats obtenus lors de la précédente étape incite à envisager un élargissement du champ d'attention : une étude complète de la lecture dans les textes de littérature personnelle doit aussi tenir compte des citations, des références aux auteurs, aux textes ou aux personnages, et enfin des critiques.

Cela est vrai dans le cadre d'une étude de la lecture d'écrivains. L'abondance de références littéraires dans leurs écrits personnels est directement liée à leur activité professionnelle. Un tel corpus ne peut cependant être considéré comme représentatif de l'ensemble de la littérature personnelle, et, en ce sens, notre étude se distingue de l'une des préoccupations communes de l'histoire de la lecture, qui s'attache à reconstituer les pratiques des lecteurs « ordinaires ${ }^{23}$ ». Ce parti pris réagit au postulat, cher aux études littéraires, voulant que l'écrivain construit plus ou moins consciemment à l'intérieur de ses textes la figure d'un lecteur idéal qui de- 
vrait être vu comme porteur d'indications sur la bonne manière de déchiffrer son texte. À ce lecteur idéal des poéticiens ${ }^{24}$, dont on pourrait critiquer l'" ethnocentrisme lettré ${ }^{25}$ ", l'histoire de la lecture tend à opposer le lecteur ordinaire, dont les compétences variables, mais surtout la liberté, transcendent les indications inscrites dans le texte. Les deux disciplines occultent le fait que l'écrivain emprunte lui aussi le chemin de la lecture ordinaire, à sa façon qui n'est certainement pas celle du plus grand nombre, mais que l'on peut considérer comme son modèle amplifié. Denis Saint-Jacques, dont les recherches s'intéressent plus spécifiquement à la littérature de grande consommation, le reconnaît en proposant l'étude de deux récits autobiographiques de Michel Tremblay. Dans la mesure où le dramaturge

reconstruit [sa vie] comme un enchaînement de lectures[,] [...] ce parti pris en fait un informateur exceptionnel pour une petite enquête très littéraire où un seul, auteur reconnu, est autorisé à témoigner pour tous pourvu que cela sache retenir l'attention de façon exemplaire $^{26}$.

Particulièrement typiques des textes personnels d'écrivains, les références littéraires, les citations et les critiques permettent de glaner des informations à la fois sur la lecture individuelle et sur une culture littéraire commune à des ensembles sociaux plus vastes. Elles doivent cependant être traitées avec plus de précaution que les témoignages directs concernant la lecture, car elles ne nous renseignent que sur les lectures probables ou possibles des textes ${ }^{27}$. La référence à un auteur ou la citation d'un texte suppose certes une connaissance littéraire. Celle-ci peut résulter de la lecture mais pourrait aussi être issue des études, d'une culture générale ou de l'influence des discours ambiants. Nul n'a besoin par exemple d'avoir lu le Don Quichotte de Cervantès pour faire référence 
aux personnages de ce roman et à plusieurs de ses épisodes : ceux-ci sont bien connus, leurs paroles citées, leurs aventures partout racontées. Leurs figures se meuvent dans l'horizon intellectuel de l'Occident autant qu'entre les pages du livre. Il existe une circulation du littéraire par le biais d'autres médias que le livre, ce qui rend problématique l'utilisation des citations et références comme témoins de la lecture.

La valeur des occurrences de ce type, dans le cadre d'une recherche sur les pratiques individuelles de lecture, ne va donc pas de soi. Prises isolément, les sources personnelles sont ici déconcertantes, puisque rien ne permet de dire avec certitude si tel texte mentionné a bien été lu. Trop souvent incomplètes ou incertaines, elles ne permettent pas la reconstruction du corpus lu par l'écrivain. Les travaux menés en théorie littéraire sur la citation et sur l'intertextualité sont d'un secours limité. Comme c'est le cas pour les recherches sur la représentation du livre et de la lecture dans la fiction littéraire, ils restent généralement centrés sur le texte et ne s'interrogent pas sur la lecture probable qui est à l'origine, pour l'auteur, de l'acte de citer. Lorsqu'ils considèrent la lecture, c'est au chapitre de la réception du texte et des effets de l'insertion intertextuelle sur le lecteur implicite ou idéal. La citation sert alors de clef de lecture ou d'interprétation du texte dans lequel elle s'insère ${ }^{28}$; ailleurs, elle soutient une théorie générale de la littérature comme entrecroisement de textes et de discours. Peu de chercheurs s'attachent à interpréter l'usage de la citation dans le but de comprendre comment le texte cité était lu ou compris à telle époque ${ }^{29}$, si ce n'est dans le cas d'études d'influences, orientant elles aussi leur analyse vers le texte, cette fois dans l'optique de retracer sa genèse ${ }^{30}$.

Pour établir un pont entre les références littéraires et la lecture, une étude à dominante historique ne peut demeurer fermée sur la littérature personnelle. En ce qui concerne les 
auteurs québécois, on est d'autant plus en droit de se méfier de la valeur des références et des citations comme indicateurs de la lecture, que l'enseignement classique en vigueur jusqu'à la fin des années 1960 repose largement sur l'étude de manuels et de morceaux choisis, qui «dispens[ent] de lire les œuvres, ou de les lire dans leur version intégrale ${ }^{31} »$. Ceux-ci permettent de construire un discours sur un auteur dont on n'a lu aucun texte en entier. Plusieurs textes personnels étudiés font état de ces méthodes, les critiquent, rappellent les moyens qu'ils ont dû déployer, adolescents et jeunes adultes, pour accéder aux textes ${ }^{32}$. Occultent-ils pour autant les connaissances générales ainsi acquises ${ }^{33}$ ?

Ainsi, les citations et références abondant dans la littérature personnelle d'écrivains acquièrent leur valeur lorsqu'il est possible de les jumeler à d'autres sources, telles que l'étude des manuels et programmes d'enseignement suivis par l'auteur, ou encore l'inventaire de sa bibliothèque personnelle. Les recoupements établis par de telles études multidirectionnelles permettent d'arriver à des conclusions plus appuyées et d'articuler entre eux des résultats par nature plutôt statiques. Mis face à face, un index des auteurs cités et une liste des livres possédés permettent de reconstituer un usage des textes ${ }^{34}$. L'analyse des méthodes d'interprétations proposées par les institutions d'enseignement fréquentées par un auteur pourrait préciser davantage les conditions de leur appropriation.

\section{Rhétorique et lecture}

Le problème de l'interprétation des citations et des références dans l'optique des pratiques de lecture doit par ailleurs tenir compte d'une distinction importante entre les types de textes très divers que l'on regroupe sous l'appellation de littérature personnelle. Selon l'expérience de recherche menée sur les écrits personnels d'écrivains québécois, leur usage semble 
en effet être largement tributaire de la visée du texte où elles s'insèrent. Qu'un texte soit destiné à la publication, rédigé pour soi-même ou envoyé à un ami, la fonction détermine l'orientation rhétorique du texte.

Dans le cas des textes rétrospectifs - mémoires, autobiographies et autres souvenirs entre lesquels la distinction n'est guère tranchée - - publiés ou non, il faut d'abord distinguer entre l'auteur qui choisit de présenter son parcours d'un point de vue public et celui qui s'attarde davantage à la vie privée. Lorsque le texte a une visée publique, il arrive que l'auteur s'en serve comme d'une plate-forme pour justifier et défendre des choix contestés par ses opposants, que ceux-ci soient politiques, idéologiques ou simplement personnels. Le mémorialiste, en quelque sorte, consigne sa version des faits. La référence à des textes lus se fait polémique, critique. Paradoxalement, cette littérature personnelle révèle alors une lecture professionnelle, et l'acte de lecture lui-même s'éclipse pour livrer une interprétation, une lecture du texte. Les Rencontres avec moi-même d'Hermas Bastien ${ }^{35}$ appartiennent à ce type d'écrits. Une part importante des lectures de l'auteur est attestée par le dialogue qu'il établit avec ses confrères journalistes ou écrivains. Il mentionne la lecture de leurs ouvrages au moment de leur parution et cite les passages qui justifient son approbation ou ses critiques, allant jusqu'à rectifier les affirmations de certains. Il cite par ailleurs, comme en réponse, les critiques de ses propres ouvrages ${ }^{36}$. Les Rencontres avec moimême offrent donc une riche source d'informations sur les lectures de travail d'un homme très actif dans les milieux intellectuel et universitaire montréalais du milieu du $\mathrm{XX}^{\mathrm{e}}$ siècle et sur une certaine communication entre des écrivains qui se lisent entre eux. Elles restent cependant beaucoup plus discrètes en ce qui concerne les circonstances de la lecture, et 
particulièrement des lectures de loisir, que seules quelques allusions laissent deviner.

Lorsque les textes sont écrits pour être publiés, et ce, peu importe qu'ils se concentrent sur la vie privée ou sur la vie publique, on remarque l'adoption d'une attitude rhétorique visant à la construction d'une persona d'écrivain ou de lettré. L'allusion littéraire est alors outil rhétorique ${ }^{37}$ : elle établit l'autorité de l'auteur en exhibant son érudition ou remplit une fonction d'ornementation qui vaut autant par le contenu de la référence ou de la citation que par le simple fait de citer ou de se référer. Ainsi, après quelques propos sur « la fragilité de la gloire terrestre ", Roger Duhamel observe : «Bossuet a dit tout cela beaucoup mieux que moi $^{38} »$, affichant à la fois sa connaissance du moraliste et une modestie qui suscitera la bienveillance de son lecteur. Une citation indirecte ${ }^{39}$ ou dont la source est occultée ${ }^{40}$ construit l'image d'un lettré qui manie les textes avec nonchalance. Le choix des citations et la manière de les intégrer en disent donc autant sur l'image que l'autobiographe ou le mémorialiste veut projeter que sur le contenu réel de ses lectures. En soi, cette rhétorique de la citation peut s'avérer un objet d'étude intéressant en ce qu'elle révèle les lectures valorisées ou dévalorisées par l'écrivain en plus d'indiquer en quoi il souscrit aux valeurs de son temps ou en quoi il s'en distingue. Ringuet écrit dans ses Confidences:

en vérité, je suis trop sérieux pour lire de ces romans idiots où le crime le plus parfait est toujours puni [...]. Il se trouve bien chez moi quelques douzaines de ces petits bouquins aux cartonnages bigarrés, aux titres sanglants. Je vous assure que ce sont des exemplaires oubliés par quelque ami de passage ${ }^{41}$.

En se défendant de posséder et de lire des romans policiers, le romancier indique en creux la fortune populaire de ces li- 
vres au moment de l'écriture des Confidences, énonce en tant qu'intellectuel sa désapprobation de principe... et nous laisse libres de le croire ou non lorsqu'il jure n'avoir pas lui aussi succombé au plaisir désœuvré d'en ouvrir un.

Le même principe rhétorique s'applique aux scènes de lecture et au discours sur celle-ci. Les témoignages de Victor Barbeau et de Ringuet, contemporains, ne s'accordent pas sur la disponibilité du livre à Montréal durant l'entre-deux-guerres. Selon le premier, «Montréal avait [...] un marché du livre [...] capable de satisfaire aux goûts, avoués ou secrets, de la clientèle. À vue d'œil rien ne semblait y manquer»; les livres moins avouables se trouvaient "sous le manteau ${ }^{42}$ ». Quant à Ringuet, originaire de Trois-Rivières, il dit avoir dû attendre de s'installer à Montréal avant d'assouvir sa soif de lectures. De la métropole, il rappelle néanmoins que le Fraser Institute fut le seul endroit où il put dénicher les livres refusés ailleurs : "Sans [cette maison], la connaissance de la littérature me fut apparemment restée fermée ${ }^{43}$ ». Que doit-on lire dans ce décalage ? Le simple effet du hasard ayant donné à l'un les bonnes adresses, ou l'amplification chez Ringuet d'une situation réelle qui contribue à projeter l'image de l'anticonformiste que le romancier se targue d'avoir toujours été ? La structure des Confidences, récit rétrospectif bâti comme une succession de brefs fragments articulés autour d'un thème donné, appelle la formule. Le souci de construction d'une image de soi pour le lecteur est ici clairement perceptible et doit nous inciter à la prudence. Une fois de plus, les informations sur la lecture collective fournies par les sources plus traditionnelles de l'histoire de la lecture, la reconstitution d'un discours social sur la lecture grâce à l'étude des programmes d'enseignement et des prescriptions de lecture émanant des autorités religieuses ou simplement des pages littéraires des journaux, permettraient, en s'additionnant avec l'analyse du texte per- 
sonnel, de jauger avec plus de précision la situation du lecteur singulier.

Les textes de littérature personnelle destinés à la publication présentent des points de vue sur la lecture fort différents de ceux que l'on peut tirer des correspondances ou des journaux intimes. Ces derniers répondent en effet à des fonctions autres qui prêtent aux passages témoignant de la lecture une couleur fort différente. Écrits pour consigner des faits et gestes ou dans un effort d'auto-analyse, les journaux intimes sont les plus susceptibles d'approfondir les pratiques privées de la lecture. Lorsque l'auteur prend le parti d'utiliser ses lectures comme outil d'analyse de soi, comme c'est le cas pour de Saint-Denys Garneau et Fernand Ouellette, le journal est la source d'information la plus riche ${ }^{44}$. Un autre journal, celui de Jean-Paul Desbiens ${ }^{45}$, présente sous forme de citations et de critiques un suivi au quotidien, apparemment rigoureux, des abondantes lectures de son auteur. Celles-ci servent de tremplin pour la réflexion, sans toutefois être analysées en tant que pratique, car pour Desbiens, l'engagement du lecteur n'est pas le même que celui de l'écrivain : « lire c'est consommer plus qu'on ne rend", écrit-il, alors qu'« écrire, c'est donner ${ }^{46}$ ». Si la finesse des relations établies avec les textes nous échappe, un tel journal nous permet néanmoins de déduire un corpus de livres lus et d'établir un usage introspectif du texte. Il est difficile de savoir si la richesse de certains journaux repose simplement sur un choix de composition, celui d'organiser l'écriture de soi autour des lectures, ou si à l'inverse, un tel choix découle de pratiques originales donnant à la lecture un rôle plus essentiel dans le développement de la personnalité. 


\section{Conclusion : la communauté littéraire}

Nous avons vu à quel point il est rare de pouvoir reconstituer avec précision et exactitude le portrait d'un lecteur individuel. Le problème des sources, qui se pose en général pour la discipline de l'histoire de la lecture et explique les moyens détournés utilisés pour développer un discours historique sur un objet si difficile à cerner, entrave aussi les études singulières. Pour arriver à dresser un tableau complet des pratiques de lecture d'un individu qui nous intéresse, il faudrait pouvoir consulter à la fois ses écrits intimes, ses œuvres publiées et sa correspondance, détenir un catalogue de sa bibliothèque personnelle et avoir une idée des livres offerts par les bibliothèques et librairies qu'il fréquentait, retracer les programmes d'études qu'il a suivis... Ainsi, avant même de se heurter au problème de l'articulation de ces différentes sources d'information entre elles, surgit la difficile, voire impossible tâche de les rassembler, car il est rare qu'elles soient toutes localisables pour un même individu.

L'accumulation des sources est proposée par plusieurs spécialistes de l'histoire de la lecture ${ }^{47}$, mais relativement peu d'ouvrages mettent ce principe en application ${ }^{48}$. Plusieurs collectifs présentent d'excellentes études émanant de disciplines diverses, sans toutefois permettre que les résultats de cellesci ne se croisent ${ }^{49}$. Rassembler et analyser des sources aussi diverses paraît une tâche moins ardue lorsqu'elle s'organise autour d'une communauté de lecteurs précisément circonscrite.

L'étude d'une communauté littéraire ${ }^{50}$ se révèle ainsi profitable. Par ce terme nous désignons un groupe d'individus partageant des intérêts intellectuels et qui sont effectivement en communication les uns avec les autres, que ce soit directement ou par lettres. Ils s'échangent des livres ou se les conseillent, discutent de leurs lectures, lisent et commentent les 
textes écrits par les uns et les autres s'il s'avère que la communauté compte parmi ses membres des écrivains. Le mot membre utilisé ici ne doit pas laisser penser que la communauté littéraire est nécessairement un groupe organisé. Certaines peuvent s'articuler autour d'une revue - on peut penser, dans les années 1930, au groupe de La Relève ${ }^{51}$ — ou d'un groupe politique, comme l'Action canadienne-française dont fait partie Hermas Bastien. D'autres peuvent avoir pour centre un lieu de rencontre, comme l'Arche de la Tribu des Casoars, un grenier où se réunit un groupe de jeunes assoiffés de culture et dont Ringuet rappelle le souvenir ${ }^{52}$. On limiterait cependant leur portée si on ne tenait pas compte d'un réseau plus diffus, s'appuyant sur l'existence d'une communication (centrée autour des lectures et de la culture) entre les individus. Pour accéder à une compréhension meilleure de l'acte de lecture et des conditions dans lesquels il s'effectue, on recherchera en effet les signes d'un dialogue, lequel mot peut désigner aussi bien les échanges entre un écrivain et son éditeur que les conseils ou impressions de lecture circulant entre amis. Les individus qui constituent une communauté littéraire auront souvent en commun une formation semblable, puisque de telles communautés tendent à se former à l'époque des études ${ }^{53}$. Ils partagent donc un bagage culturel semblable, ce qui - c'est notre hypothèse - ne peut qu'influencer leurs manières de lire. Non seulement une partie de leur fond littéraire, de leur bibliothèque intérieure, sera semblable, mais les notions d'analyse littéraire ou plus simplement de morale qu'ils ont acquises en un même lieu d'étude orienteront leur lecture, même lorsqu'ils tentent de s'en distinguer.

La notion de communauté littéraire serait donc apparentée à celle de réseaux de sociabilité intellectuelle ou littéraire ${ }^{54}$. Toutes deux peuvent recouvrir des réalités diverses 
allant des relations privées jusqu'aux associations semi-professionnelles ou professionnelles - une diversité qui correspond à celles des manières de lire.

Une forme de la littérature personnelle jusqu'ici laissée de côté, les correspondances supposent une relation de communication entre deux individus et répondent en cela à des visées bien distinctes des textes rétrospectifs destinés à la publication et des journaux intimes. L'échange entre deux correspondants à propos des lectures révèle un usage situé à michemin entre la lecture solitaire et les pratiques collectives que sont, par exemple, les lectures effectuées en classe. Elles contribuent à établir une communauté littéraire, c'est-à-dire une relation au centre de laquelle se situe la lecture ${ }^{55}$.

L'étude d'une communauté littéraire permet en outre de pallier aux inévitables "trous" dans les sources. Tel membre du groupe n'a pas laissé ses écrits intimes, mais on possède l'inventaire de sa bibliothèque personnelle; tel autre n'inscrit dans ses œuvres de fiction que peu de représentations de la lecture mais a produit de nombreux textes critiques; un autre encore est prolifique sur les effets de ses lectures tout en restant allusif quant aux titres lus, alors qu'un correspondant nous fournit les informations manquantes; et ainsi de suite. Par ailleurs, l'intégration des œuvres de fiction à l'étude d'une communauté littéraire paraît essentielle. On ne peut en effet se couper de la fiction sous prétexte qu'elle n'offre pas un reflet fidèle du réel. Les personnages de romans lisent, citent et mentionnent des œuvres littéraires bien réelles; en cela, la fiction, si elle ne peut être considérée comme un témoignage, s'inscrit dans le réel, elle y puise ses problèmes, elle s'y adresse ${ }^{56}$. Au même titre que la littérature personnelle, il faut considérer l'aspect rhétorique de la fiction et s'en servir pour l'interroger, pour la faire parler. 
En considérant d'entrée de jeu, et en théorie, la communauté littéraire comme un seul individu et en cumulant les sources, on pourrait obtenir une image de la lecture qui soit moins floue et ainsi, plus révélatrice au point de vue de l'histoire intellectuelle. En restant par ailleurs, tout au long de l'entreprise, attentif aux singularités de chaque individu et aux désaccords qui ne laisseront pas d'exister entre eux, il serait possible d'éviter les généralisations stériles et de dégager de l'ensemble une série de portraits de lecteurs qui s'éclairent entre eux. De cette manière, rendre justice au caractère éminemment individuel de l'acte de lecture et faire apparaittre l'une de ses dimensions collectives seraient à la portée des recherches sur la lecture.

\section{NOTES}

'Sophie Montreuil, « Bibliographie internationale de l'histoire de la lecture", dans Yvan Lamonde et Sophie Montreuil, dir., Lire au Québec au XIX' siècle, Montréal, Fides, 2003, pp. 285-306. La section consacrée aux études sur les pratiques individuelles de lecture tient sur trois pages, alors que celle recensant les travaux sur les pratiques collectives en occupe plus de dix. Le mérite d'avoir établi la distinction entre les pratiques de lecture collectives et individuelles, essentielle au point de vue méthodologique, revient à Y. Lamonde et S. Montreuil dans l'introduction du même ouvrage, « Pour une histoire des pratiques de lecture : éléments de méthode et pacte fondateur », pp. 7-16.

${ }^{2}$ On trouvera une série d'articles dressant des bilans de la recherche sur la lecture aux États-Unis et dans plusieurs pays d'Europe (Italie, Espagne, Allemagne, Hollande, Angleterre et Russie) dans Roger Chartier, dir., Histoires de la lecture, un bilan des recherches : actes du colloque des 29 et 30 janvier 1993, Paris, Paris, IMEC/Maison des sciences de l'homme, 1995, 320 p.

${ }^{3}$ On émet certes des hypothèses concernant les comportements de lecteurs singuliers, à partir par exemple des connaissances établies sur l'objet matériel qu'est le livre, ou alors à partir des textes visant à contrôler la lecture. Celles-ci demandent cependant à être vérifiées. 
${ }^{4}$ Roger Chartier, L'ordre des livres, Aix-en-Provence, Alinéa, 1992, p. 13. Yvan Lamonde et Sophie Montreuil soulignent aussi l'intangibilité de la lecture comme objet de recherche. Voir « Pour une histoire des pratiques de lecture [...] », dans Y. Lamonde et S. Montreuil, dir., op. cit., p. 8.

${ }^{5}$ À titre d'exemple, on peut penser au modèle proposé par Rolf Engelsing, selon lequel le développement de l'industrie de l'imprimerie a donné lieu à une révolution de la lecture : «a "reading revolution" occurred in this period in which the manner of reading shifted from the "intensive" and repeated scrutiny of a few texts to the "extensive" and cursory reading of many" (R. Engelsing, Der Bürger als Leser : Lesergeschichte in Deutschland, 1500-1800, Stuttgart, Kohlhammer, 1971, cité par John Brewer, «Reconstructing the Reader : Prescriptions, Texts and Strategies in Anna Larpent's Reading », dans James Raven, Helen Small et Naomi Tadmor, dir., The Practice and Representation of Reading in England, Cambridge, Cambridge University Press, 1996, p. 243). Ce modèle, contesté par plusieurs pour son manque de nuance, témoigne néanmoins de manière convaincante des variations historiques du rapport aux textes (pour un état de ces critiques et pour d'autres arguments en faveur du caractère historique de la lecture, voir David Hall, « Les lecteurs et la lecture dans l'histoire et dans la théorie critique. Un exposé sur la recherche américaine ", dans Roger Chartier, dir., Histoires de la lecture [...], op. cit., pp. 173-176).

"Voir Robert Darnton, « La lecture rousseauiste et un lecteur "ordinaire" au XVIII" siècle ", dans Roger Chartier, dir., Pratiques de la lecture, Marseille, Rivages, 1985, pp. 126-155, un texte d'abord paru en anglais dans R. Darnton, The Great Cat Massacre and Other Episodes in French Cultural History, New York, Vintage Books, 1984, 298 p.

${ }^{7}$ John Brewer, loc. cit.

${ }^{8}$ Valentin Jamerey-Duval, Mémoires. Enfance et éducation d'un paysan au XVIII siècle, présentés par Jean-Marie Goulemot, Paris, Le Sycomore, 1981, 423 p. Ces mémoires ont servi à une étude menée par Jean Hébrard : " Comment Valentin Jamerey-Duval apprit-il à lire ? L'autodidacte exemplaire », dans Roger Chartier, dir., Pratiques de la lecture, op. cit., pp. 24-60.

"Voir Frédéric Hardel et Yvan Lamonde, « Lectures domestiques, d'exil et de retraite de Louis-Joseph Papineau (1823-1871) ", dans Y. Lamonde et S. Montreuil, dir., op. cit., pp. 19-67 ; Y. Lamonde, « La lecture et "le livre de l'histoire" chez Amédée Papineau (1835-1845) ", dans Ibid., pp. 69-93; F. Hardel, "Lectures de Lactance Papineau, un "Canadien malheureux" (18351845) ", dans Ibid., pp. 95-121; et S. Montreuil, «(Se) lire et (se) dire : José- 
phine Marchand-Dandurand et la lecture (1879-1886) ", dans Ibid., pp. 123150.

${ }^{10} \mathrm{Ce}$ projet, qui avait pour but l'évaluation des possibilités de ces sources autant que leur analyse, a eu lieu durant l'été 2003 à l'Université McGill dans le cadre du projet « History of the Book in Canada/Histoire du livre et de l'imprimé au Canada " (HBiC/HLIC), sous la direction de Yvan Lamonde assisté de Sophie Montreuil. Le corpus comprenait des textes de Louis Fréchette, Philippe Panneton (Ringuet), Victor Barbeau, Hermas Bastien, Hector de Saint-Denys Garneau, Roger Duhamel, Gérard Bessette, Jean-Paul Desbiens et Fernand Ouellette.

${ }^{11}$ Ces remarques méthodologiques sont issues d'un second assistanat de recherche mené à l'été 2004 à l'Université McGill, toujours sous l'égide du projet $\mathrm{HBiC} / \mathrm{HLIC}$.

${ }^{12}$ Hector de Saint-Denys Garneau, Journal, texte conforme à l'édition critique établie par Giselle Huot, Présentation de Wilfrid Lemoine, Montréal, Bibliothèque Québécoise, 1996, 477 p. ; Idem, Lettres à ses amis, Montréal, $\mathrm{HMH}$, 1967, 489 p. ; Idem, "Correspondance ", dans CEurres, texte établi, annoté et présenté par Jacques Brault et Benoît Lacroix, Montréal, Presses de l'Université de Montréal, 1971, pp. 757-1045.

${ }^{13}$ Fernand Ouellette, Journal dénoué : essai, Montréal, l'Hexagone, 1988 [1974], 263 p. (coll. « Typo »).

${ }^{14}$ La manière de lire de ces deux poètes fera l'objet d'une publication séparée.

${ }^{15}$ Louis Fréchette, Mémoires Intimes, deuxième édition, texte établi et annoté par Georges A. Klinck, préface de Michel Dassonville, Montréal, Fides, 1977, $206 \mathrm{p}$.

${ }^{16}$ Victor Barbeau, La tentation du passé, Ottawa, Éditions La Presse, 1977, pp. 63-87.

${ }^{17}$ Roger Duhamel, Bilan Provisoire, Montréal, Beauchemin, 1958, pp. 47-48.

${ }^{18}$ " Je fais un exercice de mémoire : sans consulter ma bibliothèque, $j$ 'essaie de me dire qui sont mes auteurs préférés, par ordre alphabétique. [...]" (JeanPaul Desbiens, Se dire, c'est tout dire (journal), Montréal, L'Analyste, 1989, p. 99, repris dans Journal d'un bomme farouche, Montréal, Boréal, 1993, p. 108).

${ }^{19}$ " De tous les romans que j’ai lus, je n'en retiens que trois : Voyage au bout de la nuit ; Joumal d'un curé de campagne; L'bomme sans qualités. Et dans cet ordre. J'ajouterais la femme du pauvre et Poil de Carotte. Terminé ! Je ne négocie plus. » (J.-P. Desbiens, Les années novembre, Montréal, Éditions Logiques, 1996, p. 357) 
${ }^{20}$ Philippe Panneton, Journal de Ringuet, présenté par Francis Parmentier et Jean Panneton, Montréal, Guérin, 1998, 334 p.

${ }^{21}$ Francis Parmentier, «Présentation » dans Ibid., p. vi (l'auteur souligne).

${ }^{22}$ Ibid., p. 186 (Chateaubriand), pp. 254-256 (Dante), pp. 103 et 133 (Huysmans).

${ }^{23}$ Cette préférence pour la lecture populaire est notamment le fait d'un groupe de recherche du Centre de recherche en littérature québécoise (CRELIQ, aujourd'hui remplacé par le Centre de recherche interuniversitaire sur la littérature et la culture québécoises [CRILCQ]) qui s'intéresse à la réception des best-sellers au Québec (voir Denis Saint-Jacques, dir., L'Acte de lecture, Québec, Nuit blanche, 1994, 305 p.) ; elle se fait aussi sentir dans les travaux de Roger Chartier, notamment sur la Bibliothèque Bleue, une collection de livres bon marché dans la France d'Ancien Régime (R. Chartier, "Du livre au lire », dans R. Chartier, dir., Pratiques de la lecture, op. cit., pp. 62-90 ; Idem, Lectures et lecteurs dans la France d'Ancien Régime, Chapitres II, VII et VIII, Paris, Seuil, 1981, 370 p.), et dans ceux de Robert Darnton sur Jean Ranson (loc. cit.). Cette préoccupation pour les lecteurs populaires pourrait dériver des recherches en sociologie de la littérature, amorcées au début des années 1970 autour de Robert Escarpit, qui étudie la littérature du point de vue de la communication de masse (voir Nicole Robine, "La lecture ", dans R. Escarpit, dir., Le Littéraire et le social: éléments pour une sociologie de la littérature, Paris, Flammarion, 1970, pp. 221-244).

${ }^{24}$ Nous faisons ici référence au large courant des théories de la réception, fondé sur les travaux de Hans Robert Jauss (Pour une esthétique de la réception, Paris, Gallimard, 1978, 305 p.) et de Wolfgang Iser (L'acte de lecture. Théorie de l'effet esthétique, Bruxelles, Mandarga, 1985, 405 p.).

${ }^{25}$ L'expression est de Gérard Mauger, Claude F. Poliak et Bernard Pudal, "Introduction générale », dans Histoires de lecteurs, Paris, Nathan, 1999, pp. 923. Les pages introductives de cette étude de psychologie gagnent à être lues pour les mises au point méthodologiques qu'elles établissent. Les auteurs critiquent les théories de la lecture dont « l'analyse reste focalisée sur le sens du texte » et non sur la lecture : «la pratique de la lecture est confondue avec la pratique professorale du commentaire. [...] elle prête aux lecteurs "ordinaires" un regard herméneutique et esthète $[. .$.$] qui leur est, sauf exceptions,$ étranger [...]» (Ibid., p. 15).

${ }^{26}$ Denis Saint-Jacques, «Présentation », dans D. Saint-Jacques, dir., op. cit., p. 14. 
${ }^{27}$ Selon le " pacte d'interprétation des sources sur la lecture " proposé par Yvan Lamonde et Sophie Montreuil, il convient d'interroger « le degré de réalité de la lecture tel qu'inféré de la source qui en rend l'étude possible ». Quatre degrés sont ainsi proposés : "lecture réelle, probable, possible ou fictive » (Y. Lamonde et S. Montreuil, loc. cit., pp. 10-12 [les auteurs soulignent]).

${ }^{28}$ Cette tendance herméneutique est la plus répandue dans les travaux sur la lecture en études littéraires. Les représentations de la lecture, notamment dans la fiction, sont étudiées en tant que lieux du texte où s'énoncent les règles devant mener à sa lecture idéale. Encore une fois, leur objet d'étude reste le texte littéraire, et non la lecture. Le collectif dirigé par Jan Herman et Paul Pelckmans, L'Épreuve du lecteur. Livre et lectures dans le roman d'Ancien Régime, Louvain-Paris, éditions Peeters, 1994, 498 p., est exemplaire de cette approche.

${ }^{29}$ Voir l'article d'Élisabeth Nardout-Lafarge, « La mise en scène de la référence littéraire chez Hertel et Lemelin ", Études françaises, vol. 29, no 1 (printemps 1993), pp. 77-96. L'auteur y interprète de façon historique le mode d'intégration des citations au texte littéraire. Les études sur la citation se penchent très généralement sur les textes de fiction.

${ }^{30}$ L'étude de Roland Bourneuf, Saint-Denys Garneau et ses lectures européennes, Québec, Presses de l'Université Laval, 1969, 332 p., est l'une d'elles. Si la visée de cette étude ne porte pas l'auteur à s'intéresser de prime abord aux façons de lire, on y trouve malgré tout d'intéressantes observations sur celles-ci, notamment sur la façon dont le poète lit et interprète les textes à la lumière de son expérience personnelle. On peut aussi noter l'article de Jean-Pierre Lapointe, "Sur la piste américaine : le statut des références littéraires dans l'œuvre de Jacques Poulin ", Voix et images, vol. XV, nº 1 (43) (automne 1989), pp. 15-27, qui porte sur les influences littéraires américaines du romancier de Volkswagen blues.

${ }^{31}$ Max Roy, «La valorisation des objets littéraires », dans Joseph Melançon, Nicole Fortin et Georges Desmeules, dir., La Lecture et ses traditions, Québec, Nuit blanche, 1994, p. 123. Cette étude " abord[e] la question de la lecture à l'école » en se penchant sur des devoirs d'élèves de Belles-Lettres dans les séminaires et collèges classiques (Ibid.,p. 124) en regard des méthodes d'enseignement de l'époque.

32 « [...] de la littérature française, de la vraie, je ne connaissais que dates apprises par cœur dans notre Manuel de littérature, jugements... conditionnés et maigres morceaux choisis " (Ringuet, Confidences, Montréal/Paris, Fides, 1965, p. 151). Voir aussi Jean-Paul Desbiens, Sous le soleil de la pitié, Montréal, 
Éditions du Jour, 1965, p. 29 ; Idem, Journal d'un homme farouche, op. cit., pp. 28 29 ; Hermas Bastien, Rencontres avec moi-même, Bibliothèque Nationale du Québec, Fonds MSS-257, boîte 1, p. 159.

${ }^{33}$ Sur l'éducation classique au Québec et sa position par rapport à la lecture, plusieurs études éclairent les conditions dans lesquelles nos auteurs ont d'abord accédé aux textes : Jean Cinq-Mars, Histoire du College Sainte-Marie de Montréal, 1848-1969, Montréal, Hurtubise HMH, 1998, 516 p. ; Joseph Melançon, Clément Moisan et Max Roy, Le discours d'une didactique : la formation littéraire dans l'enseignement classique au Québec (1852-1967), Québec, Centre de recherche en littérature québécoise (CRELIQ), Université Laval, 1988, 456 p. (coll. « Recherche », n” 1) ; Simone Vannucci, « La critique littéraire des jésuites dans la revue Collège et famille, 1944-1960 : construire une élite », dans Josée Vincent et Nathalie Watteyne, dir., Autour de la lecture. Médiations et communautés littéraires, Québec, Nota bene, 2002, pp. 131-146.

${ }^{34}$ L'article d'Annie-Claude Prud'homme, " "Voyage autour d'une bibliothèque" : la littérature dans les catalogues de bibliothèques personnelles d'écrivains (1880-1910) ", dans Yvan Lamonde et Sophie Montreuil, dir., op. cit., pp. 159-200, propose une telle étude croisée « des citations dans l'œuvre de Faucher de Saint-Maurice, en parallèle avec l'analyse de son catalogue » (Ibid., p. 184). Plus loin, elle met en parallèle le catalogue de Jules Fournier et Souvenirs de prison, pamphlet où ce polémiste révèle « ses préoccupations de lecteur » (Ibid., p. 186), en plus de convoquer pour son analyse les prises de positions du journaliste concernant la littérature. Il s'agit d'un bel exemple d'article où les différentes sources s'appuient les unes sur les autres pour dessiner le portrait d'un lecteur.

${ }^{35}$ Hermas Bastien, op. cit. Docteur en philosophie puis en pédagogie, professeur, il est auteur de plusieurs ouvrages de philosophie, de pédagogie et de littérature et fut actif tout au long de sa carrière au sein de plusieurs revues dont Le Quartier Latin, L'Action universitaire, La Revue dominicaine, L'Action française, L'Action nationale et l'Information médicale et paramédicale.

${ }^{36}$ Il fait ainsi état de lectures de livres de Lionel Groulx, son interlocuteur le plus important (Ibid., pp. 175-176, 279-280 et 323-325), de ceux de Guy Frégault (Ibid., p. 316), Jean Bruchési (Ibid., p. 307), Esdras Minville (Ibid., pp. 272273), pour n'en nommer que quelques-uns.

${ }^{37}$ Voir Stephan Morawski, "The Basic Functions of Quotations ", dans A.-J. Greimas et al., Sign Language, Function, La Haye, Mouton, 1970, pp. 690 705, cité par Elisabeth Nardout-Lafarge, loc. cit., p. 80. 
${ }^{38}$ Roger Duhamel, op. cit., p. 152.

${ }^{39}$ Par exemple : « Le scolaire Boileau prétendait que l'ennui nait de l'uniformité » (Ibid., p. 172).

${ }^{40}$ Dans les Confidences, Ringuet s'adresse à ses lecteurs et cite sans la nommer la Chanson de Roland: « Rappelez-vous vos classiques : "Charlemagne, l'empereur à la barbe fleurie" " (Ringuet, op. cit., p. 175).

${ }^{41}$ Ibid., pp. 81-82.

${ }^{42}$ Victor Barbeau, op. cit., p. 64.

${ }^{43}$ Ringuet, op. cit., p. 154.

${ }^{44}$ Contrairement à ce qu'on a vu avec le Journal de Ringuet, les lectures sont alors analysées en elles-mêmes. Fernand Ouellette écrit à propos de ses lectures de Dostoïevski : "Je vivais intensément avec tous les personnages du Russe. Je devenais ces personnages. [Ils] devinrent mes compagnons quotidiens, plus réels que les hommes que je côtoyais au bureau. [...] Grâce à Dostö̈evski, je m'humanisais par personnes interposées » (F. Ouellette, op. cit., pp. 42-43).

${ }^{45}$ Le journal de Jean-Paul Desbiens est publié régulièrement depuis 1983, d'abord sous forme de fragments dans la revue L'Analyste, rassemblés en volume dans Se dire, c'est tout dire (op. cit.), puis intégralement à partir de 1993. Journal d'un bomme farouche (op. cit.) reprend les années 1983-1992 ; les livraisons suivantes paraitront tous les deux ou trois ans.

${ }^{46}$ Jean-Paul Desbiens, Se dire, c'est tout dire, op. cit., p. 88.

${ }^{47}$ Voir notamment Robert Darnton, « First Steps Toward a History of Reading ", Australian Journal of French Studies, XXIII, 1 (1986), pp. 5-30.

${ }^{48}$ Roger Chartier utilise des sources variées pour Lectures et lecteurs dans la France d'Ancien Régime (op. cit.), une collection d'articles réunis en volumes. Au nombre de ses sources se trouvent quelques textes personnels, notamment les Mémoires de V. Jameray-Duval (Ibid., pp. 223-224) et des correspondances (Ibid., pp. 207-209, pp. 224 et suiv.).

${ }^{49}$ C'est le cas de collectifs publiés au cours des dix dernières années au Québec : Denis Saint-Jacques, dir., op. cit.; Joseph Melançon, Nicole Fortin et Georges Desmeules, dir., op. cit. ; Josée Vincent et Nathalie Watteyne, dir., op. cit.

${ }^{50}$ Le terme est emprunté à James Smith Allen, dans un article intitulé « Une république des lettres romantique. Marie-Sophie Leroyer de Chantepie et sa 
communauté littéraire, 1836-1876 ", dans Graham Falconer, dir., Autour d'un cabinet de lecture, Toronto, Centre d'études du XIX ${ }^{e}$ siècle Joseph Sablé, 2001, pp. 55-67. L'auteur désigne par cette expression le « libre d'échange d'idées entre les auteurs [...] et le public» (Ibid., p. 57) qui caractérise selon lui le romantisme français, et plus précisément, en prend pour exemple la relation épistolaire établie entre Leroyer, tenancière de cabinet de lecture, écrivain et lectrice, et ses auteurs favoris, George Sand et Gustave Flaubert. La réalité décrite par M. Allen, qu'il est à notre avis fécond de transposer au-delà de l'époque étudiée dans son article, correspond à «la conscience [des écrivains] d'appartenir à une communauté littéraire formée de réseaux d'amitié entre écrivains (ou entre écrivains et lecteurs) " (Ibid., p. 58).

${ }^{51}$ Sur La Relève comme lieu de socialisation littéraire, voir l'étude de Nancy Houle : « La Relève : une revue, un réseau », dans Pierre Rajotte, dir., Lieux et réseaux de sociabilité littéraire au Québec, Québec, Nota bene, 2001, pp. 113-153.

${ }^{52}$ Ringuet, op. cit., pp. 51-57.

${ }^{53}$ Dans le cas du groupe gravitant autour de La Relève, la plupart ont fréquenté le Collège Sainte-Marie. Il faut cependant se garder de limiter la communauté littéraire à un groupe d'anciens étudiants d'une institution donnée, suivant ainsi la même logique qu'en ce qui concerne les groupes d'appartenance organisés.

${ }^{54}$ Autour de laquelle s'organise le recueil de Pierre Rajotte, dir., op. cit.

${ }^{55}$ Les réseaux de correspondance constituent une autre forme de sociabilité littéraire identifiée dans le recueil dirigé par Pierre Rajotte. Voir l'article de Richard Giguère, «Sociabilité et formation des écrivains de l'entre-deux-guerres. Le cas des réseaux de correspondance d'Alfred DesRochers », dans Pierre Rajotte, dir., op. cit., pp. 35-69.

${ }^{56}$ Sur la " réalité » des bibliothèques romanesques, voir Nathalie Ferrand, Livre et lecture dans les romans français du XVIII siècle, Paris, Presses Universitaires de France, 2002, pp. 242-252 : « rien de plus réel que les bibliothèques dans les romans du XVIII siècle, dont les titres authentiques ancrent la fiction dans la réalité contemporaine et dans [l'] actualité éditoriale [...]» (Ibid., p. 246). Le roman québécois du milieu du $\mathrm{XX}^{\mathrm{e}}$ siècle utilise lui aussi fréquemment la référence à des titres réels. André Belleau remarque cette légion de références littéraires et culturelles dans les romans de Jean Simard, Jacques Godbout, Réjean Ducharme et Yolande Villemaire ("Code social et code littéraire dans le roman québécois ", L'Esprit créateur, vol. XXIII, n" 3 (automne 1983), pp. 27-28). 